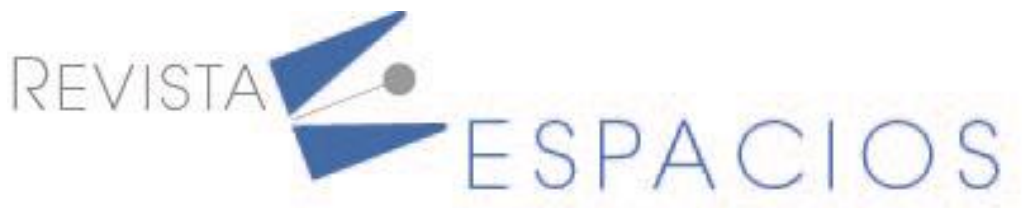

Vol. 41 (43) $2020 \cdot$ Art. 13

Recibido/Received: 31/07/2020 • Aprobado/Approved: 28/08/2020 • Publicado/Published: 12/11/2020

\title{
Solución al problema de la selección de medidas de seguridad industrial dentro una empresa usando inteligencia artificial
}

\section{Applied Intelligence artificial for the solution to the problem of the selection of safety measures in a company}

\author{
ESPITIA IBARRA, Mateo ${ }^{1}$ \\ PLAZA GÓMEZ, Maria T. ${ }^{2}$ \\ HERNANDEZ RIAÑO, Helman E. ${ }^{3}$
}

\begin{abstract}
Resumen
Es innegable que la seguridad industrial se traduce en un factor de éxito para las empresas que la adoptan en su operación. Este estudio presenta una alternativa factible para selección de medidas de seguridad industrial en las empresas. Se emplea un modelo de mochila multicriterio, solucionado a través de dos algoritmos genéticos con buen rendimiento en calidad de solución y tiempo de computación.

Palabras clave: seguridad Industrial, medidas de seguridad, metaheurísticas, algoritmo genético.

Abstract

Certainly the industrial security it traslate in a success fact for the companies that adopt it in their operation. This study identify a feasible alternative for the safety industrial measures selection in the companies. A multicriteria knaspack problem is used, solved through two genetic algorithms with a good performance regarding to solution and computation time.

key words: metaheuristics, genetic algorithm, safety measures selection.
\end{abstract}

\section{Introducción}

Hoy en día la economía mundial y la lucha por la disminución del desempleo recae sobre el empresarismo y la creación de empresas (Banco Interamenicano de Desarrollo, 2000). Ello ha significado que cada día sean creados más puestos y perfiles de trabajos, que siempre están acompañados por una serie de peligros particulares, los cuales deben ser gestionados para su mitigación, eliminación o reducción dependiendo la capacidad económica de cada empresa para lograrlo. Hay que considerar que en muchos casos las partidas de prepuesto para medidas de seguridad industrial son insuficientes e incluso inexistentes para una adecuada gestión de los riesgos laborales. Por parte de las empresas existe la creencia que invertir en medidas de seguridad industrial es irrelevante o infructuoso, sin embargo, lo cierto es que ello es una apreciación irreal de los riesgos de accidentes,

\footnotetext{
${ }^{1}$ Departamento de Ingeniería Industrial, Universidad de Córdoba, carrera 6 No. 76-103, Montería, Colombia, mespitiaibarra@gmail.com.

${ }^{2}$ Departamento de Ingeniería Industrial, Universidad de Córdoba, carrera 6 No. 76-103, Montería, Colombia, mtplaza@correo.unicordoba.edu.co

${ }^{3}$ Departamento de Ingeniería Industrial, Universidad de Córdoba, carrera 6 No. 76-103, Montería, Colombia, hhernandez@correo.unicordoba.edu.co
} 
y esta percepción usualmente se produce porque hay serias dificultades para medir, evaluar y controlar el riesgo (Bianchini et al., 2016).

Es habitual encontrar estudios que dictan las pautas para evaluar, medir e implementar medidas que optiman las condiciones de seguridad en los centros de trabajo, también se pueden ubicar herramientas para determinar la eficiencia del Sistema de Gestion de Seguridad y Salud en el Trabajo (SG-SST), aunque usualmente se basan en indicadores como la relación costo/beneficio, subestimando el impacto que pudiese tener una medida sobre otra. En este último caso, el trabajador es quien resulta perjudicado porque aumenta su exposición, al encontrarse en un ambiente laboral copado de medidas de seguridad que se interponen entre ellas mostrando sus deficiencias funcionales. Todo esto puede influir en las percepciones del riesgo, en las reglas, en los procedimientos afectando directamente la seguridad de estos y por ende la efectividad del sistema de gestión de seguridad (Shezeen et al., 2018).

Es innegable que la seguridad industrial se traduce en un factor de éxito para cualquiera empresa que la adopte en su operación, como disciplina de la gestión organizacional brinda muchas herramientas para prevenir, estudiar y evitar los accidentes de trabajo y enfermedades laborales, hecho que se refleja en la minimización de costos relacionados al absentismo laboral. Por ello las empresas consientes con la seguridad laboral no solo se han enfocado en mejorar sus procedimientos para ser más productivos y competitivos, sino también en mejorar los sistemas de seguridad y salud en el trabajo, buscando situaciones factibles de inversión y beneficio.

Gopang et al., (2017) sintetizan a partir de encuestas sobre medidas de salud y seguridad ocupacional y su efecto sobre el desempeño de pequeñas y medianas empresas a través de análisis estadísticos que existe correlación positiva moderada entre la implementación correcta de las medidas de salud y seguridad ocupacional y el desempeño de las empresas. Con ello reafirmamos la premisa que una implementación adecuada de las medidas de seguridad ocupacional, conllevan a un mejor desempeño empresarial, particularmente en pequeñas y medianas empresas, según el estudio mencionado al principio del párrafo, además, ello puede contribuir significativamente a su éxito y crecimiento.

Un estudio exploratorio realizado en centros de petroquímicos de Malasia buscaba determinar la influencia entre factores de evaluación de prácticas de seguridad. Considerando una serie de indicaciones de International Atomic Energy se desarrolló un modelo alternativo de nueve (9) factores de evaluación de prácticas de seguridad en los que se incluyen: actitud de cuestionamiento, información comunicativa, entorno laboral, compromiso de la gerencia, comunicación, prioridad de seguridad, visión personal, implicación y enfoque prudente. El estudio sobre los factores que afectan las prácticas de seguridad describió que el factor más significativo fue la gestión de la seguridad y el entorno de trabajo. Además, agregaron que el entorno de apoyo y la excelente comunicación sobre salud y seguridad con el objeto de garantizar que las personas de la organización estén informadas puede entregar resultados muy positivos para la empresa (Hamid et al., 2018).

Por otro lado, Stolzer y otros ( 2018) se enfocaron en desarrollar una métrica para determinar la eficiencia del sistema de medidas de seguridad en las organizaciones a nivel individual, resaltaron el hecho de que la implementación de un sistema de medidas de seguridad se traduce en una inversión inmensa para una organización, hecho por el que al momento de precisar en qué se va a invertir se deberían tener una serie criterios fiables que la soporten. Los autores realizaron un análisis envolvente de datos para cada uno de los cuatro componentes del sistema de medidas de seguridad. Primero, se recopilaron las entradas y salidas, seguidamente se les realizo un tratamiento para ser examinados y preparados según sea necesario. Las entradas incluyeron preguntas de escala Likert pidiendo a los encuestados que identifiquen cómo sus organizaciones implementaron el sistema de medidas de seguridad en las cuatro áreas componentes: política y objetivos de seguridad, gestión de riesgos de seguridad, garantía de seguridad y promoción de la seguridad. 
El avance de las tecnologías de información y las ciencias computacionales han repercutido en todas las áreas del conocimiento; la seguridad industrial no ha sido ajena a este fenómeno, hecho que ha supuesto un cambio en la forma de como abarcar esta disciplina, enfocándola desde una nueva alternativa al incluir metodologías como la optimización exacta, optimización aproximada e inteligencia artificial, estableciendo alternativas cuantitativas que mejoran la eficiencia de las medidas de seguridad, con consideraciones claras de estas últimas en cuanto a las oportunidades y deficiencias que podrían suponer.

En su estudio Caputo et al., (2013), muestran con un enfoque cualitativo el desarrollo de un algoritmo para optimizar la inversión en medidas de seguridad para una planta de producción de tamaño mediano. También Choi y Loh (2017) muestran en su estudio un modelo de control industrial, que buscaba atenuar los efectos de los accidentes de trabajo en la República de Corea, concluyendo que los accidentes de trabajo solo pueden mitigarse mediante la aplicación de medidas preventivas que mengüen la probabilidad de ocurrencia, además, consideran que situaciones de riesgos con bajas probabilidades de ocurrencia también deben tomarse medidas de acción, todo desde el punto de vista económico, social y psicológico.

El trabajo de Caputo et al., (2011) es un ejemplo de la descripción de la dificultad que supone la asignación de recursos para el sistema de seguridad y salud en el trabajo, los autores abordan el problema mediante una función objetivo que considera a la seguridad industrial desde el ámbito económico y lo solucionan empleando un algoritmo genético. En ese estudio se muestra una solución lógica para desentrañar una situación compleja de la gerencia de riesgos laborales, allí se caracterizan diversos escenarios donde se podría aplicar el método descrito mediante la optimización de recursos direccionados a seleccionar medidas de seguridad teniendo en cuenta el musculo financiero disponible, contemplando para este último tanto variables estocásticas como determinísticas.

Otro estudio que se enfoca desde el costo de oportunidad, la inversión en materia de seguridad industrial por parte de las empresas y el gobierno, nos muestras dos escenarios principales: el costo de escases debido a un accidente de trabajo y el costo excesivo de inversión de acuerdo a los niveles de riesgo que se encuentren en la organización (Ma et al., 2016). En dicho trabajo se buscaba mejorar la inversión y a su vez minimizar el costo de oportunidad perdido frente a los costos reales que se pueden presentar en la empresa en materia de seguridad. Se concluyo que el nivel de inversión óptimo del gobierno y las empresas en temas de seguridad crece directamente con la escasez de la unidad de costos, y por el contrario disminuye con el aumento del costo de exceso de unidad, además, la decisión de inversión de la empresa de seguridad muestra que la inversión obligatoria de seguridad acrecentará el costo de oportunidad esperado e inversiones de seguridad de una empresa.

Dicho enfoque puede no resultar muy ajustado a la realidad organizacional porque tal como lo tratan de explicar, las medidas de seguridad impactan los indicadores de seguridad y económicos, además, pueden intervenir en la efectividad de una u otra medida (Abrahamsen et al., 2018).

En adición a la falta de metodologías rigurosas para establecer medidas de seguridad, tenemos que para este proceso hay ausencia de un carácter propio de cada empresa, característica que es muy importante dado que establece el rango de inversión que es capaz de asumir la organización para el sistema de gestión de seguridad y salud en el trabajo, que a fin de cuentas es el factor principal al momento de escoger dichas medidas y minimizar los riesgos dentro de la organización. Este estudio puede presentar a las empresas, una alternativa robusta y factible para la toma de decisiones en torno al sistema de seguridad y salud en el trabajo, buscando mejorar su estructura financiera y su desarrollo integral para que ello se traduzca en ventajas competitivas con el objeto de hacer crecer a la empresa y alcanzar sus metas continuamente. 


\section{Metodología}

Se empleo una metodología no experimental-transeccional-exploratoria debido al hecho de que la investigación parte de un área del conocimiento que, pese a haber desarrollado avances hace más de una década, recién comienza a ser abarcado con aplicaciones alternativas de las metodologías de optimización aproximada a problemas y temas propios de la administración científica y la gestión de organizaciones como lo es el sistema de gestión de seguridad y salud en el trabajo. El diseño de investigación "No Experimental", se empleó porque no hubo manipulación deliberada de variables y solo se observó un fenómeno en su ambiente natural para después analizarlo.

\subsection{Modelo matemático.}

Se propone emplear un modelo de mochila multicriterio conforme a la particularidad del problema de elección de medidas dentro de un sistema de seguridad y salud en el trabajo. Este problema se aborda desde un modelo matemático que congrega distintos aspectos de formulación como lo son la multiplicidad de factores de seguridad requeridos por la legislación vigente y las limitaciones económicas. Se usa el modelo matemático propuesto por Caputo, Pelagagge y Salini (2013) para la elección de las medidas de seguridad (SM por sus siglas en inglés) dentro de una empresa. En dicho modelo, cada una de las medidas de seguridad $x_{i}\left(x_{1}, x_{2}, \ldots x_{n}\right)$ candidatas a ser elegidas son representadas con variable binaria de acuerdo a la decisión tomada, es decir, $x_{i}=1$ si la medida de seguridad i-ésima es escogida, $x_{i}=0$ de lo contrario. La Figura 2 ilustra la dinámica de selección de las medidas de seguridad dentro del modelo de optimización.

Figura 1

Dinámica de selección de las medidas de seguridad

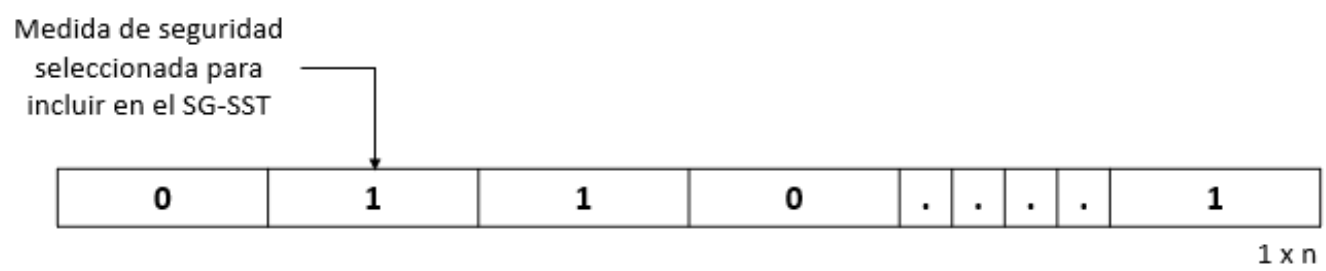

Fuente: Elaboración Propia.

El criterio de optimización y restricciones se exponen, a continuación:

$\operatorname{Max} \sum_{\mathrm{i}=1}^{\mathrm{n}} \mathrm{x}_{\mathrm{i}} \mathrm{U}_{\mathrm{i}}$

Sujeto a:

$$
\begin{aligned}
& \sum_{i=1}^{n} x_{i} C_{i} \leq C_{\text {max }} \\
& \sum_{j=1}^{n} x_{j} C M_{i j}<1, \forall i \\
& x_{i} \geq x_{j} \\
& x_{i} \geq O b_{i}, \forall i \\
& R=p M
\end{aligned}
$$


En el modelo planteado la función objetivo (1) se encarga de maximizar la utilidad $U_{i}$ asociada a la medida de seguridad $x_{i}$. La ecuación (2) relativa al costo de las medidas de seguridad, donde el conjunto de $n$ medidas de seguridad son asociadas a un costo $C_{i}$ que representa el costo de la medida de seguridad $i$ en las mismas unidades monetarias en las que se exprese el capital máximo $\left(C_{\text {max }}\right)$. La ecuación (3) expresa la compatibilidad entre medidas de seguridad, pues existen los casos donde las medidas son incompatible entre sí, por ejemplo, implementar una medida $\mathrm{X}$ imposibilita usar la medida Y. Por ello se define una matriz de compatibilidad (CM) $n^{*} n$ donde $C M_{i j}=0$ cuando la medida $i$ es compatible con la medida $j$ y $C M_{i j}=1$ en el caso contrario. Cabe resaltar que obviamente, $C M_{i i}=0$ para todo $i$. Así entonces, con la introducción de la matriz de compatibilidad $C M$, para cada medida de seguridad $i$ debe probarse que no se haya escogido dentro de la misma solución una medida incompatible $j$. La ecuación (4) representa ya sea la dependencia o la precedencia de una medida de seguridad sobre otra, es decir, que una medida necesite la existencia de otra medida de seguridad en la organización o una medida de seguridad deba ser ejecutada una vez se haya aplicado otra con anterioridad. La ecuación (4) refiere a la obligatoriedad de una medida ya sea por cuestiones legales u otras consideraciones, así $O b_{i}$ hace referencia al valor $i$ dentro de la matriz de obligatoriedad de las $n$ medidas de seguridad. $O b_{i}=1$ si la medida de seguridad $i$ es obligatoria y $O b_{i}=0$ en el caso contrario.

Hay que aclarar que la utilidad empleada en el modelo es resultado de una puntuación evaluada bajo seis criterios: efectividad, costo, eficiencia, rango, aplicabilidad y funcionalidad (Caputo, 2008). En ese sentido la efectivad es la capacidad de una medida de seguridad de reducir significativamente el riesgo asociado a un peligro, entonces el nivel de riesgo asociado a un peligro se define como la probabilidad de accidente $p$, multiplicado por la magnitud de las consecuencias $M$, tal cual como se expresa en la ecuación (6). Dicha probabilidad y magnitud de consecuencias se expresan de acuerdo a la Tabla 1.

Tabla 1

Parámetros de clasificación de riesgo

\begin{tabular}{|c|c|c|c|c|c|}
\hline $\begin{array}{l}\text { Clasificación de } \\
\text { probabilidad }(p)\end{array}$ & Nivel & $\begin{array}{c}\text { Frecuencia de } \\
\text { ocurrencia de } \\
\text { referencia }\end{array}$ & $\begin{array}{l}\text { Clasificación } \\
\text { Nivel de } \\
\text { gravedad (M) }\end{array}$ & Nivel & $\begin{array}{l}\text { Valor de perdida } \\
\text { de referencia }\end{array}$ \\
\hline 5 & Frecuente & $>10^{-1}$ & 5 & Catastrófico & $>10$ muertes \\
\hline 4 & Probable & $10^{-1}-10^{-2}$ & 4 & Crítico & 10 más muertes \\
\hline 3 & Ocasional & $10^{-2}-10^{-3}$ & 3 & Pertinente & $\begin{array}{l}\text { Fatalidad } \\
\text { ocasional }\end{array}$ \\
\hline 2 & Remoto & $10^{-3}-10^{-4}$ & 2 & Marginal & Lesiones mayores \\
\hline 1 & Improbable & $<10^{-5}$ & 1 & Despreciable & Lesiones leves \\
\hline
\end{tabular}

Fuente: Caputo 2013.

Los criterios de costo, eficiencia y rango son evaluados en una escala de 1 a 5 como se describen en la Tabla 2. La aplicabilidad hace referencia a la practicidad en la implementación de la medida de seguridad, ello significa que no implique interrupciones en actividades productivas y se pueda adaptar a los requerimientos, estructuras y equipamientos existentes de la organización. Por último, la funcionalidad se enfoca en la posibilidad de que la interacción de la medida de seguridad con los procesos y trabajadores sea positiva. 
Tabla 2

Parámetros de clasificación

de costo, eficiencia y rango

\begin{tabular}{|c|c|c|c|c|c|}
\hline & \multicolumn{5}{|c|}{ Escala } \\
\hline & 1 & 2 & 3 & 4 & 5 \\
\hline Costo Anual & Despreciable & Bajo & Promedio & Pertinente & Muy alto \\
\hline Eficiencia & $\begin{array}{l}\text { La SM afecta } \\
\text { principalmente } \\
\text { a los peligros } \\
\text { más críticos }\end{array}$ & $\begin{array}{l}\text { La SM afecta } \\
\text { principalmente } \\
\text { algunos peligros } \\
\text { relevantes }\end{array}$ & $\begin{array}{c}\text { La SM afecta } \\
\text { principalmente a } \\
\text { los peligros que } \\
\text { tienen criticidad } \\
\text { media }\end{array}$ & $\begin{array}{c}\text { La SM afecta } \\
\text { principalmente a } \\
\text { peligros de baja } \\
\text { criticidad }\end{array}$ & $\begin{array}{l}\text { La SM afecta } \\
\text { principalmente a } \\
\text { peligros que tienen } \\
\text { una importancia } \\
\text { despreciable }\end{array}$ \\
\hline Rango & $\begin{array}{l}\text { La SM afecta } \\
\text { virtualmente a } \\
\text { todos los } \\
\text { peligros } \\
\text { existentes }\end{array}$ & $\begin{array}{l}\text { La SM afecta a la } \\
\text { mayoría de los } \\
\text { peligros } \\
\text { existentes en la } \\
\text { instalación }\end{array}$ & $\begin{array}{l}\text { La SM afecta } \\
\text { múltiples peligros } \\
\text { existentes en la } \\
\text { instalación }\end{array}$ & $\begin{array}{l}\text { La SM solo } \\
\text { afecta a unos } \\
\text { pocos peligros } \\
\text { existentes en la } \\
\text { instalación }\end{array}$ & $\begin{array}{l}\text { La SM se centra en } \\
\text { un solo peligro } \\
\text { existente en la } \\
\text { instalación }\end{array}$ \\
\hline
\end{tabular}

Fuente: Caputo 2013.

\subsection{Metaheurística}

De acuerdo a los antecedentes en la literatura se seleccionó la metodología denominada "algoritmo genético" (genetic algorithm) para la solución del problema de optimización, se realizaron adecuaciones conforme a las características particulares de la selección de medidas de seguridad. El algoritmo genético está conformado por un grupo de fases durante su desarrollo, que fueron inspiradas por el fenómeno de la selección natural (Soft Computing y Sistemas de Información Inteligentes, 2014), a continuación describimos cada una de ellas.

Inicialización: El primer paso es generar una población inicial con un tamaño poblacional ( TP ) de soluciones factibles generadas aleatoriamente que deben cumplir íntegramente las restricciones del modelo de optimización. Para la elaboración de las soluciones iniciales se emplea un enfoque de construcción, este garantiza que las soluciones generadas respeten las condiciones para asegurar su factibilidad, el único criterio de rechazo es la restricción de costo, por lo que el costo de la combinación de una posible solución se encuentre dentro del presupuesto asignado para el sistema de seguridad y salud en el trabajo.

Selección: En esta etapa se escoge cierta cantidad de soluciones propuestas de acuerdo al rendimiento de cada una de las posibles soluciones. Para este caso se utilizan operadores de cálculo del valor de la función objetivo y se escogen al mejor $50 \%$ de las soluciones iniciales, para así conformar el conjunto de "padres" que tendrá la posibilidad de pasar sus genes a la siguiente generación y el $50 \%$ de las soluciones restantes se eliminan como parte del proceso de muerte de individuos (Soft Computing y Sistemas de Información Inteligentes, 2014). En este estudio se adaptó el método de selección por torneo binario, es decir, se escogen dos padres de forma aleatoria del conjunto de soluciones y se compara su rendimiento en la función objetivo, y se escoge al mejor de ellos dos, en ese sentido el conjunto de padres tendría un tamaño de $T P / 2$ soluciones.

Cruzamiento: Con el conjunto de padres conformado prosigue el cruzamiento, operación basada en el cruzamiento genético de la reproducción sexual de los seres de la naturaleza (Artificial, 2012). Primero se establece una probabilidad de cruzamiento, ella indica la probabilidad que tiene un padre de transferir sus "genes" o componentes a una solución "hijo" que resulta de la combinación de los genes de dos padres. Se empleo un cruzamiento de punto para la combinación de los genes, donde se toma un punto de corte dentro de la longitud de la solución de manera aleatoria y se ubican los genes como lo indica la Figura 2. El cruzamiento se realiza hasta generar una población de soluciones descendientes del mismo tamaño de la población inicial, es decir, de tamaño TP. 
Figura 2

Operador de cruzamiento basado en un punto

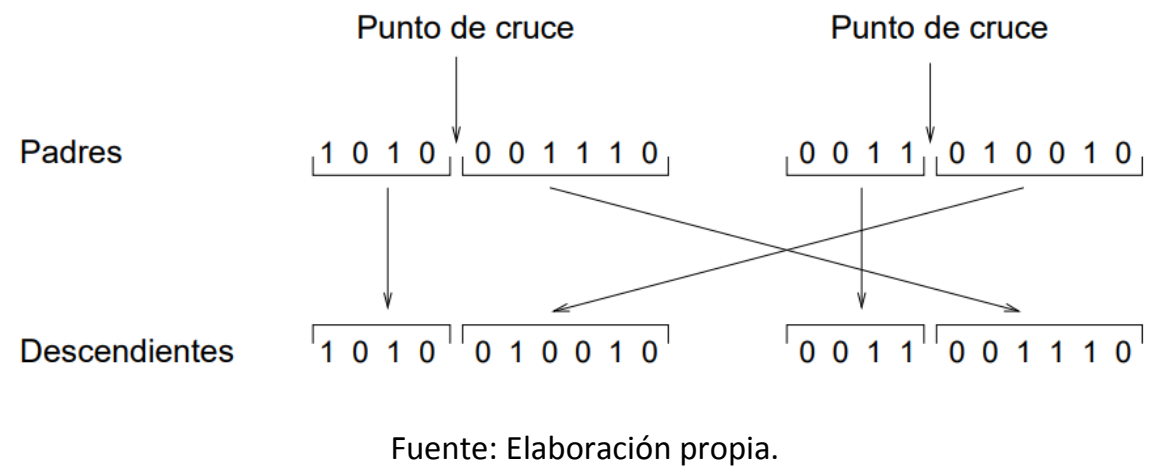

Mutación: A través de este procedimiento se modifican en una pequeña parte de la población, uno o varios genes de acuerdo a una probabilidad preestablecida por el autor, buscando una mejor explotación del espacio de soluciones factibles. Este proceso de ilustra en la Figura 3.

Figura 3

Operador de mutación

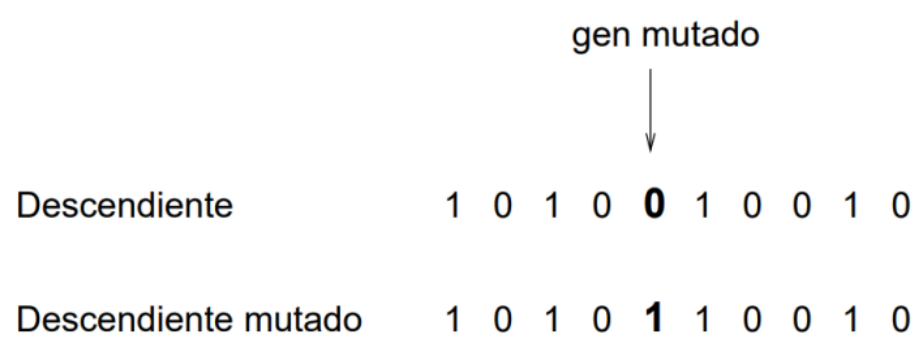

Fuente: Elaboración propia.

Reemplazo: Este operador evalúa todas las soluciones descendientes y selecciona la mejor solución encontrada en la generación actual con la mejor de generaciones previas, de manera tal que, al finalizar el número de generaciones establecidos por el usuario, se obtenga la mejor solución encontrada hasta el momento. También con este operador se actualizan en cada iteración general la población de padres, de manera tal que los descendientes de una generación se convierten en los padres de la siguiente.

\subsection{Instancia}

La instancia seleccionada fue propuesta por Pisinger (2005) en que el autor muestra instancias en gran medida estructuradas, que facilitan la evaluación del rendimiento de ciertos algoritmos de solución para el problema de la mochila. Así pues, se seleccionan como elementos pertenecientes una instancia, considerando que para ingresar a la "mochila" los elementos deben presentar buena correlación entre la utilidad y el costo, además, elementos del modelo de optimización como los matices de obligatoriedad, compatibilidad y precondición, inherentes al problema de selección de medidas de seguridad tratado en la presente investigación, fueron agregados a la instancia en concordancia con los criterios descritos en el modelo matemático. La Figura 4 es un gráfico de dispersión que permite observar la relación entre el costo y la utilidad obtenida. 
Figura 4

Gráfico Utilidad vs Costo - Instancia

medianamente correlacionada

Gráfico de Utilidad vs Costo

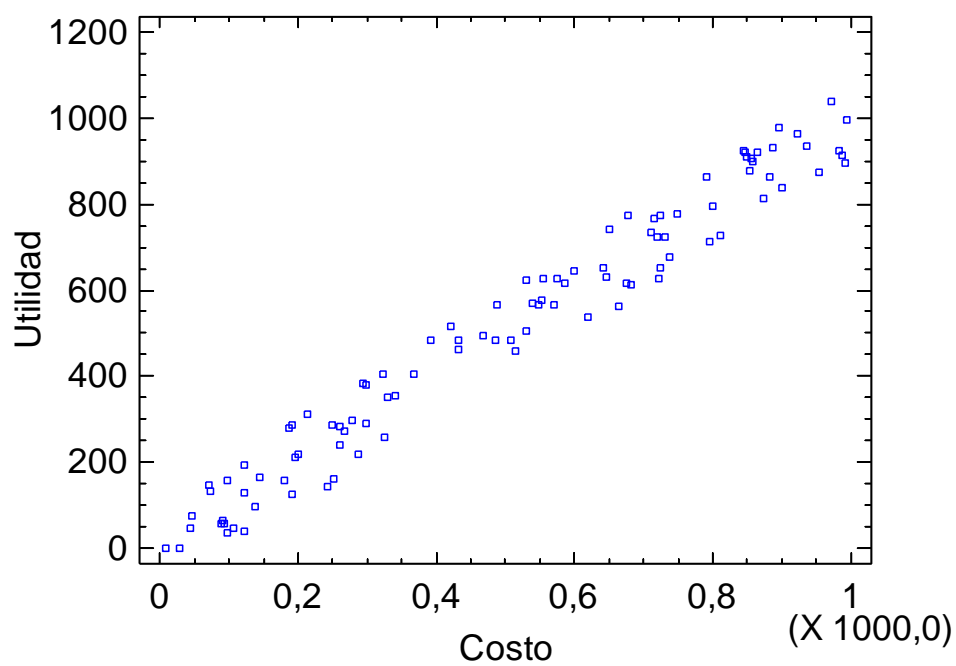

Fuente: Elaboración propia.

\subsection{Experimento}

Para probar el modelo se desarrollaron dos versiones del algoritmo genético, ellos difieren en la forma que exploran y explotan el espacio de solución del problema. Finalmente, se prueba la eficiencia de una metodología de solución basada en inteligencia artificial a través de un experimento simple. Los algoritmos utilizados tenían los siguientes parámetros: probabilidad de cruzamiento (pc) de 95\%, probabilidad de mutación (pm) $10 \%$ y tamaño de población (TP) de 100 y 800 individuos. Los parámetros utilizados para los algoritmos genéticos son el resultado de la experimentación del autor.

Posteriormente, cada algoritmo fue ejecutado sobre la instancia referida anteriormente en 30 réplicas cada una de 25 minutos. Para realizar el experimento se utilizó Python 3.6 para la codificación y ejecución de los algoritmos, en una computadora con un procesador Intel(R) Xeon(R) CPU E3-1225 v5, de 8GB RAM y sistema operativo Windows 10 Pro de 64 bits. Para el análisis de los resultados obtenidos a partir del experimento computacional se utilizó el software estadístico Statgraphics Centurion Version 18.1.06 (64-bit) bajo licencia de uso académico.

\section{Resultados}

Los resultados obtenidos por dos versiones distintas del GA, desarrolladas por los autores se presentan en la Tabla 3. A partir de los datos obtenidos, se realiza una prueba de hipótesis a fin de inquirir, mediante un diseño de experimento simple, si existen diferencias significativas entre los resultados conseguidos a través de los algoritmos. 
Tabla 3

Resultados del experimento

\begin{tabular}{cccccc}
\hline Replica & GA_1 & GA_2 & Replica & GA_1 & GA_2 \\
\hline $\mathbf{1}$ & 6979 & 6980 & 16 & 6973 & 6936 \\
$\mathbf{2}$ & 6959 & 6953 & 17 & 6955 & 7004 \\
$\mathbf{3}$ & 6892 & 6925 & 18 & 6977 & 6955 \\
$\mathbf{4}$ & 6944 & 6913 & 19 & 7008 & 6952 \\
$\mathbf{5}$ & 7004 & 6960 & 20 & 6933 & 6958 \\
$\mathbf{6}$ & 6990 & 6926 & 21 & 6968 & 6955 \\
$\mathbf{7}$ & 6969 & 6982 & 22 & 6949 & 6937 \\
$\mathbf{8}$ & 6959 & 6979 & 23 & 6998 & 6956 \\
$\mathbf{9}$ & 6987 & 6937 & 24 & 6973 & 6960 \\
$\mathbf{1 0}$ & 6970 & 6931 & 25 & 6981 & 6935 \\
$\mathbf{1 1}$ & 7004 & 6926 & 26 & 6975 & 6941 \\
$\mathbf{1 2}$ & 6953 & 6983 & 27 & 6990 & 6970 \\
$\mathbf{1 3}$ & 6979 & 6882 & 28 & 6971 & 6911 \\
$\mathbf{1 4}$ & 6998 & 6963 & 29 & 6973 & 6921 \\
$\mathbf{1 5}$ & 6968 & 6963 & 30 & 6961 & 6976 \\
\hline
\end{tabular}

Fuente: Elaboración propia

Se efectuó la prueba del estadístico $W$ de Shapiro-Wilk con los datos obtenidos en las dos muestras, para establecer si los datos cumplen con el supuesto de normalidad, en este aspecto, el P-value fue de 0,0260452 y 0,860753 para GA_1 y GA_2 respectivamente, por lo cual se puede inferir que la muestra GA_1 no proviene de una distribución normal. Por ello se emplea la prueba no paramétrica W de Mann-Whitney para comparar las medianas de dos muestras. Para este caso se prueban las hipótesis mostradas en (7).

$$
H_{0}: M_{G A_{-} 1}=M_{G A_{-} 2} \text { vs } H_{0}: M_{G A_{-} 1}>M_{G A_{-} 2}
$$

Esta prueba se construye combinando las dos muestras con los datos ordenados de menor a mayor y confrontando los ranqueos promedio de las dos muestras en los datos combinados. Debido a que el valor-P es menor que 0,05 , la mediana de la primera muestra (GA_1) es significativamente mayor que la mediana de la segunda (GA_2), con una confianza del 95,0\%, se infiere que el algoritmo genético (GA_1) presenta un mejor desempeño que el algoritmo genético (GA_2), en cuanto a la calidad de soluciones obtenidas sobre el problema planteado.

Cabe resaltar que las dos versiones del algoritmo genético desarrollados en este estudio fueron construidas a partir de la versión más sencilla de esta metodología. Sin embargo, se modificaron los procedimientos de generación de soluciones iniciales, cruzamiento y mutación.

La versión denominada GA_1 efectúa una inicialización mediante la construcción de soluciones factibles a partir de las restricciones del modelo de optimización, ello implica que cada una de las soluciones iniciales generadas es automáticamente factible. Igualmente, ejecuta el proceso de cruzamiento valiéndose de un operador de punto de corte único. Finalmente, antes de realizar la mutación se calcula un parámetro aleatorio que determina cuantas posiciones de la solución van a ser modificadas.

Por su parte la versión GA_2, para el primer conjunto de soluciones se generan soluciones de naturaleza completamente aleatorias y se conservan únicamente las que cumplan con todas las restricciones del modelo matemático. Tras esto, el proceso de cruzamiento se realiza a través de una lógica de doble punto de corte, donde se inserta la parte central de uno de los padres a la solución descendiente y el resto de esta última, viene dado por parte del segundo padre seleccionado. Por último, el proceso de mutación se desarrolla con punto 
único de mutación, es decir, se muta un solo elemento de la solución el cual es escogido aleatoriamente entre todas las posiciones de la misma y se cambia su valor manteniendo presente siempre las restricciones.

\section{Conclusiones}

En este estudio se expuso un problema de mochila multicriterio para la optimización de la asignación de recursos dentro de un sistema de seguridad, se propuso un modelo que maximiza la utilidad de cada medida de seguridad candidata, la cual está compuesta de seis criterios: eficacia, costo, eficiencia, rango, aplicabilidad y funcionalidad. El modelo fue resuelto mediante dos versiones de algoritmo genético, evaluando su desempeño en una instancia formulada. Además, por la naturaleza combinatoria del problema advirtieron las ventajas que suponen emplear algoritmos de inteligencia artificial pues generan soluciones de calidad en un tiempo computacional razonable comparada con heurísticas y/o estrategias de aproximación exacta.

El algoritmo genético forma soluciones de calidad sobre el problema en estudio, así mismo se concluye que el algoritmo genético (GA_1) genera mejores soluciones que la versión (GA_2). En síntesis, esta metaheurística adaptada es una buena alternativa para optimización de la asignación de recursos en sistema de seguridad conforme a la selección de medidas de seguridad.

Futuras investigaciones podrían orientarse en el desarrollo de modificaciones del modelo matemático para darle una perspectiva más integral de la realidad de las empresas, incluso formulando el problema con un modelo multiobjetivo basándose en variables que consideren factores que influyen en la toma de decisiones. También, la inclusión de nuevas metaheurísticas y validación de las misma en instancias grandes del problema.

\section{Referencias bibliográficas}

Abrahamsen, E. B., Moharamzadeh, A., Abrahamsen, H. B., Asche, F., Heide, B., \& Milazzo, M. F. (2018). Are too many safety measures crowding each other out? Reliability Engineering and System Safety, 174(February), 108-113. https://doi.org/10.1016/j.ress.2018.02.011

Artificial, I. (2012). Algoritmos geneticos. Algoritmos Genticos.

Banco Interamenicano de Desarrollo. (2000). Apoyo a la pequeña y mediana Empresa.

Bianchini, A., Donini, F., Pellegrini, M., \& Saccani, C. (2016). An innovative methodology for measuring the effective implementation of an Occupational Health and Safety Management System in the European Union. Safety Science.

Caputo, A. C. (2008). A novel set of ranking indices for effective selection of safety measures. 26th International System Safety Conf. (ISSC 2008).

Caputo, A. C., Pelagagge, P. M., \& Salini, P. (2013). A multicriteria knapsack approach to economic optimization of industrial safety measures. Safety Science, 51(1), 354-360. https://doi.org/10.1016/j.ssci.2012.08.002

Choi, G. H., \& Loh, B. G. (2017). Control of Industrial Safety Based on Dynamic Characteristics of a Safety Budget-Industrial Accident Rate Model in Republic of Korea. Safety and Health at Work, 8(2), 189-197. https://doi.org/10.1016/j.shaw.2016.11.002

Gopang, M. A., Nebhwani, M., Khatri, A., \& Marri, H. B. (2017). An assessment of occupational health and safety measures and performance of SMEs: An empirical investigation. Safety Science, 93(2017), 127-133. https://doi.org/10.1016/j.ssci.2016.11.024 
Hamid, M. D., Emami, S. D., Danaee, M., Che Hassan, C. R., \& Kasim, H. (2018). Determination of factors affecting safety practices in Malaysian radiation facilities. Safety Science, 104, 70-80.

https://doi.org/10.1016/j.ssci.2017.12.031

Pisinger, D. (2005). Where are the hard knapsack problems? Computers and Operations Research, 32(9), 22712284. https://doi.org/10.1016/j.cor.2004.03.002

Shezeen, O., Rudia, N., \& Kwangsu, M. (2018). La influencia del clima de seguridad, el liderazgo de seguridad, la carga de trabajo y las experiencias de accidentes sobre la percepción del riesgo: un estudio de los trabajadores coreanos de la industria manufacturera. Show.

Soft Computing y Sistemas de Información Inteligentes. (2014). Bioinformática. Algoritmos Genticos.

Stolzer, A., Friend, M., Truong, D., Tuccio, W., \& Aguiar, M. (2018). Measuring and evaluating safety management system effectiveness using Data Envelopment Analysis. Safety Science, 104, 55-69.

Esta obra está bajo una Licencia Creative Commons Attribución-NoCommercial 4.0 International

\section{$(\mathrm{cc})$ EY-NO}

